\title{
Targeting chromatin
}

Corrected: Publisher Correction

\author{
Chromatin-associated proteins are emerging as new drug targets for a variety of diseases, especially cancer. \\ This special Focus issue highlights various approaches for 'targeting chromatin' for therapeutic intervention.
}

B eyond radiotherapy, the conventional standard of care for cancer has usually been chemotherapy. Most chemotherapeutic agents target all rapidly dividing cells, although some degree of specificity may result from the inability of many cancer cells to efficiently repair DNA damage. Harm to healthy tissue leads to chemotherapy's well-known, severe side effects. Therefore, drug-development efforts have shifted increasingly toward the targeting of cancer-specific pathways, aiming at drugs specific for proteins that are mutant, overexpressed or otherwise dysregulated in cancer cells. Initial efforts focused on signaling proteins, such as kinases, which have druggable chemical features that allow the design of small molecule inhibitors. Despite remarkable initial success, this approach has encountered setbacks, including the rapid emergence of drugresistant tumor cells. Drug resistance is due in part to compensating mutations or the activation of alternative cancerpromoting pathways. This issue led to a continuing search for other drug targets, and chromatin-based mechanisms have emerged as attractive candidates.

Chromatin packages the eukaryotic genome and thereby determines the transcriptional state and the identity of a cell. Modulating the activity of chromatinmodifying proteins or proteins that 'read' the chromatin state can influence many growthrelated pathways and their intrinsic tumorsuppressor checks simultaneously, making it harder for tumor cells to develop resistance. Although no chromatin-modulating drugs have yet entered the clinic, many have shown promising results in pre-clinical and clinical studies. In this issue, we present a special Focus on Targeting Chromatin for therapeutic intervention, highlighting specific classes of chromatin-associated proteins that are the subject of current drug-development efforts, as well as other potential chromatin-related targets for which research is still at an early stage.

Husmann and Gozani (https:// doi.org/10.1038/s41594-019-0298-7) review the functions of histone-lysine methyltransferases in health and disease. Histone methylation, as well as other covalent modifications, such as acetylation, phosphorylation and ubiquitylation, has been linked to dynamic changes in chromatin structures. These modifications generally occur within the flexible $\mathrm{N}$-terminal histone tails that extend from the nucleosome core and regulate the accessibility of chromatin to trans-acting factors. Thus, methyltransferases influence a wide variety of chromatin-based processes, including transcription, replication and DNA repair, and they are potential anticancer drug targets. Inhibitors of EZH2, a histone methyltransferase that targets Lys27 of histone $\mathrm{H} 3$ and is a member of the Polycomb group of transcriptional repressors, are currently progressing through clinical trials.

Arguably the most-advanced epigenetic drugs target bromodomains. The early development of inhibitors of the bromodomain and extra-terminal class of bromodomains that showed enticing antiinflammatory and anti-cancer activities in fact boosted the idea of targeting chromatin-based processes. As discussed in a Review by Zaware and Zhou (https:// doi.org/10.1038/s41594-019-0309-8), bromodomains are acetyl-lysine 'reader' modules. By interacting with specific acetylated histones, bromodomain proteins are able to detect different chromatin states and recruit transcriptional effector proteins. Bromodomains are often paired with other domains and are present in proteins with diverse biological roles, leading to the authors' proposal of a function-based classification of these proteins.

This success of bromodomain inhibitors prompted further interest in the development of drugs that target non-bromodomain chromatin readers, as highlighted in a Perspective by Arrowsmith and Schapira (https://doi.org/10.1038/s41594-019-0290-2). These reader domains include the Royal family, plant homeodomain, WD40 repeat and YEATS domains, and the authors discuss recent progress in the discovery of small molecules that antagonize their functions. Although achieving robust cellular responses with the currently available inhibitors to these reader domains remains challenging, this constitutes a promising avenue for future research.

In their Perspective, Tzelepis, Rausch and Kouzarides (https://doi.org/10.1038/ s41594-019-0312-0) discuss recent insights into the co-transcriptional roles of RNA modifications. Although research in this area is still at an early stage, there are clear connections between changes in the so-called 'epitranscriptome' and disease phenotypes, especially a role in the development and maintenance of various types of cancer. Pharmacological targeting of RNA-modifying enzymes has not yet been achieved, but it is highly anticipated, as indicated by growing commercial interest in this area (https://doi. org/10.1038/nbt1218-1123). There is still a need to expand unbiased and quantitative assessment of RNA modifications, and recent developments, such as sequencecontextual analysis of RNA modifications by mass spectrometry, have shown promise in this context.

Mass spectrometry-based technologies are crucial to identifying the full target spectrum of active molecules and have become an indispensable step of the drugdiscovery process, as discussed by Noberini and Bonaldi (https://doi.org/10.1038/ s41594-019-0279-x), who focus on the context of epigenetic drug discovery. Whether target-based or phenotypic drug-discovery approaches are taken, it is important that all cellular targets of a potential drug be determined, not only for the identification possible off-target effects but also for potential alternative applications for a drug.

Writing in a Comment, Baskin and Haynes (https://doi.org/10.1038/s41594019-0299-6) propose that the engineering of synthetic reader-effectors that are recruited to specific chromatin features might provide an opportunity for correcting widespread gene dysregulation in cancer. The fusion of histone-binding domains from chromatin effector proteins to transcriptional regulators might allow the epigenetic reprogramming of cancer cells, although various technical limitations must still be overcome before such an approach can be considered a viable option for cancer treatment.

Despite the many challenges that remain, targeting chromatin for therapeutic intervention offers great promise for the future and provides another example of the crucial role of basic research in structural and molecular biology in driving medical and technological progress. We hope that the examples provided in this Focus issue will be an interesting read and thank all the authors for their contributions.

Published online: 3 October 2019 https://doi.org/10.1038/s41594-019-0315-X 